\title{
CARTA SOCIAL EUROPEA Y CONVENIO EUROPEO DE DERECHOS HUMANOS: PERSPECTIVAS PARA LA PRÓXIMA DÉCADA*
}

\author{
POR \\ JEAN-FRANÇOIS AKANDJI-KOMBE \\ Catedrático en la Universidad de Caen (Francia). \\ Coordinador de la Red Académica Europea sobre derechos sociales \\ (Consejo de Europa)
}

\section{CONSIDERACIONES INTRODUCTORIAS}

De entrada, debe recordarse que hay un dato a tener siempre en mente, cuando se aborda la Carta Social Europea, tanto al considerarla con entidad propia como desde el punto de vista de sus relaciones con los demás instrumentos europeos, a saber: que dicho instrumento fue concebido desde su origen en relación estrecha con el Convenio Europeo de Derechos Humanos (en adelante, CEDH). Los trabajos preparatorios dan fe sobradamente de ello. Baste aquí por tanto traer a colación dos manifestaciones significativas. La primera cabe hallarla en el dictamen emitido por la Asamblea Parlamentaria del Consejo de Euro-

* Me he permitido preparar para su publicación un texto lo más fiel posible a la presentación oral que realicé con motivo del $100^{\circ}$ aniversario de la firma de la Carta Social revisada. La principal consecuencia de ello redunda en la inclusión de referencias doctrinales y jurisprudenciales poco numerosas. [Traducción del original francés por Luis Jimena Quesada; Universitat de València] 
pa en septiembre de 1953 sobre la elaboración de la Carta Social. Se subraya en ella que el texto a elaborar «debería constituir en el ámbito social un homólogo del CEDH». La segunda emana del Comité de ministros. En el mensaje especial que éste hizo público en mayo de 1954, en el cual se fijaba el mandato del Comité social intergubernamental encargado de redactar el proyecto de Carta, se definía el instrumento que debía ver la luz como el "pariente» del CEDH en el ámbito social.

Partiendo de dicha prescripción, podría pensarse que si debía mediar alguna diferencia entre los dos textos, ella no podía sino radicar en su ámbito de intervención o su objeto: de un lado, derechos civiles y políticos y, de otro lado, derechos sociales. En cambio, las modalidades de garantía deberían ser sensiblemente idénticas, o al menos comparables.

Como es sabido, las negociaciones tendentes a la elaboración de la Carta Social Europea discurrieron en cambio por otros derroteros. El texto adoptado en Turín en 1961 no era, en lo atinente a sus modalidades de puesta en práctica y de control internacional, más que una pálida copia de su precedente de 1950. Más precisamente, el dispositivo de garantía de los derechos que debían protegerse era de los más débiles, al basarse exclusivamente en el examen de informes sometidos periódicamente a un Comité de expertos independientes por parte de los Estados; sistema que se encontraba en las antípodas del mecanismo propiamente jurisdiccional del CEDH. Más aún, dicho mecanismo de control sobre la base de informes se enfrentó inmediatamente con dificultades que prácticamente vinieron a neutralizarlo. Se comprende entonces que la Secretaria General del Consejo de Europa a la sazón, la Sra. Lalumière, con ocasión de un encuentro interregional organizado por el Consejo de Europa en enero de 1993, pudiera calificar la Carta como la "hermana pobre" del CEDH o como un "tigre de papel».

Es dicho estado de cosas el que se propuso remediar el proceso de relanzamiento, mediante una serie de instrumentos jurídicos de los cuales el más emblemático fue la Carta Social Europea revisada (en adelante, CSER o Carta revisada), firmada en 1996. Lo que nos interesa saber en esta sede es si ese texto ha podido modificar el estado de las relaciones con el $\mathrm{CEDH}$, esto es, si ha proporcionado pistas útiles que hayan hecho evolucionar esas relaciones.

A primera vista, no parece que tal haya sido el caso. En primer lugar, cabe constatar que otro era el objeto de la CSER. Se trataba de actualizar la lista de los derechos garantizados: por una parte, modernizando la problemática de algunos de ellos y, por otra parte, añadiendo derechos nuevos. Sin lugar a dudas, ello hubiera podido llevarse a 
cabo inspirándose en el CEDH y, sobre todo, en la jurisprudencia del Tribunal Europeo de Derechos Humanos. Sin embargo, es asimismo posible constatar aquí que los actores del relanzamiento se decantaron por otra vía diversa. Su fuente europea de inspiración fue el Derecho de la Unión Europea, prescindiendo de cualquier otra. En efecto, se acudió a Directivas comunitarias, en ocasiones adaptándolas, para consagrar por ejemplo el derecho de los trabajadores a la información sobre las condiciones aplicables al contrato de trabajo o a la relación laboral (artículo 2.6 CSER), el derecho de los trabajadores a la protección de sus haberes en caso de insolvencia de su empleador (artículo 25 CSER), o también el derecho de los trabajadores a la protección en caso de despido colectivo (artículo 29 CSER).

De ahí a considerar que el esquema reflejado en cada texto se había perfilado de modo inmutable como acogiendo dos sistemas paralelos, animados cada cual por una lógica de desarrollo separado, no había más que un paso. Un paso que se está tanto más tentado de franquear, desde el momento en que los órganos de control parecen suscribir dicha lógica: el Tribunal Europeo de Derechos Humanos aprovechando cuando puede la ocasión para argumentar que tal derecho está protegido por la Carta para excluirlo del campo de aplicación del CEDH -esto sucedió, en particular, en el caso del Sindicato nacional de la Policía belga (1975)—, y el Comité Europeo de Derechos Sociales recordando, en ocasiones, especialmente a los Estados que querrían eludir su obligaciones amparándose en el Convenio Europeo de Derechos Humanos, que la Carta Social y el CEDH son instrumentos distintos.

Ello no obstante, debe resistirse a semejante tentación, pues el CEDH no ha estado nunca totalmente ausente en el "paisaje" de la Carta. Según los actores y las circunstancias, el Convenio se revela ya sea como la figura de un espectro que proyecta su sombra tutelar $-y$ paralizante - ya sea como la figura de un ideal a alcanzar.

En las últimas décadas, parece haber sido esta última figura la que se ha estado imponiendo. Sobre ello volveremos. Pero en cualquier caso se toma conciencia de ello asimismo mediante diferentes proyectos y debates en torno a una posible o deseable evolución de la Carta. Por lo demás, los proyectos no son nuevos. En todo caso, dichos proyectos tienden a hacer asegurar la protección de los derechos sociales, o al menos de un cierto número de ellos, por parte del Tribunal Europeo. En defecto de ello, preconizan la transformación del Comité de derechos sociales en un Tribunal paralelo al Tribunal Europeo de Derechos Humanos. Esta alternativa fue evocada, particularmente, en las 
Recomendaciones 1354 (1998) y 1415 (1999) de la Asamblea Parlamentaria, y ha vuelto a ser retomada en los trabajos recientes del Comité director de los derechos humanos. Estos procesos institucionales, es preciso subrayarlo, deben ser inscritos en el contexto de la propia evolución del dispositivo europeo de protección de derechos humanos y en sus orientaciones de fondo que la doctrina jurídica ha sabido actualizar, a saber: de un lado, la irrupción jurisprudencial de los derechos sociales en el ámbito de los derechos humanos ${ }^{1}$, pero igualmente la formidable reticencia del Tribunal Europeo de Derechos Humanos a asumir todos los derechos garantizados por la Carta Social, pese a que incluso el desarrollo de sus técnicas y métodos de interpretación podría permitírselo².

Es precisamente esa reticencia, en la medida en que expresa límites del enfoque interpretativo, la que invita a plantear una evolución posible, reflejada en los textos, del dispositivo de protección de los derechos sociales, y la que explica los proyectos y debates mencionados más arriba. Estos últimos presentan un punto común. Se asientan en la idea de que el principio, ahora ya asumido, de la indivisibilidad de los derechos humanos, implica la unificación del régimen de los derechos $y$, por ende, de los instrumentos y mecanismos de garantía.

Aun admitiendo que esta perspectiva representa ciertamente el ideal a alcanzar, los planteamientos que siguen no van a centrarse en ella. La consignación de los derechos sociales en el CEDH, lo mismo que el establecimiento de un Tribunal Europeo de Derechos Sociales, no pueden llevarse a cabo sin una revisión de los tratados europeos de derechos humanos, opción que los organizadores de la presente celebración ya han excluido de entrada de los esquemas de evolución de la CSER en el curso de la próxima década. No vamos a discutir respecto a dicha exclusión. Puede ser, tal como se percibe en el seno del Consejo de Europa, que los Estados miembros no estén preparados para una mutación semejante, y que todavía haya que prepararlos para ello durante algún tiempo.

El caso es que siempre que pretendan extraerse pistas de consolidación de los lazos y de la profundización de las relaciones entre el

1 Véase, en particular, SUDRE, F.: «La perméabilité de la Convention européenne des droits de I'homme aux droits sociaux", Mélanges J. Mourgeon, Bruxelles, Bruylant 1998, pp. 467 ss.

2 Ver a este respecto el estimulante estudio del Profesor SUDRE, F.: «La protection des droits sociaux par la Cour européenne des droits de I'homme: un exercice de jurisprudence fiction", Rev. trim. dr. h., 2003, pp. 755 ss. 
CEDH y la Carta Social hay que estarse estrictamente a los instrumentos vigentes. Así, el ejercicio que sigue consistirá en descubrir esas pistas y explotar sus virtualidades. Se convendrá fácilmente en que una vía trazada de tal suerte es estrecha y tortuosa. Sin embargo, no por ello resulta impracticable. Sin revisar los textos, es posible efectivamente, para los órganos del CEDH y de la Carta, así como en general del Consejo de Europa, actuar en pro del desarrollo de una problemática común de garantía de los derechos (II), y de favorecer la puesta en marcha de mecanismos articuladores (pasarelas) institucionales y procedimentales entre los dos sistemas (III).

\section{EL DESARROLLO DE UNA PROBLEMÁTICA COMÚN DE GARANTÍA}

La proposición responde a una triple constatación y, más aún, a una triple evidencia. La primera radica en que la Carta y el CEDH constituyen todavía a fecha de hoy instrumentos distintos y fundan dos sistemas separados. La segunda consiste en que tanto la una como el otro están concebidos con un mismo fin, a saber, la protección efectiva de los derechos humanos. Y la tercera, que en esta materia la Carta acusa un cierto retraso con relación al CEDH. Estos elementos conducen a considerar que una manera de aproximar los dos instrumentos es hacer evolucionar el sistema de la Carta de forma que alcance un nivel de protección comparable al del Convenio. En nuestra opinión, se verifica aquí una condición necesaria para el desarrollo de las interacciones entre los dos sistemas. Para alcanzar dicho resultado, la interpretación constituye un medio ineludible. Sólo a través de ella cabría, en el estadio actual, superar algunos obstáculos que generen una problemática común.

\section{El medio: la interpretación de la Carta}

Como es sabido, el desarrollo del CEDH se asienta en buena medida en el dinamismo interpretativo del Tribunal Europeo de Derechos Humanos. Es éste el que ha propiciado una evolución de las normas extrayendo de ellas nuevas implicaciones o nuevas exigencias, haciendo así del Convenio un "instrumento de Derecho vivo". También es sabido que dicha evolución afecta al conjunto del Convenio, habiendo consistido especialmente en una extensión relativa de su ámbito de aplicación a los derechos sociales y en un desarrollo extraordinario de las garantías procesales. Casi no merece la pena recordar que esos avances 
son el resultado de un enfoque que ha combinado algunos principios interpretativos, como por ejemplo el principio de efectividad de los derechos, con técnicas que han permitido el «desarrollo lógico de los conceptos" esenciales del Convenio, técnicas entre las cuales descueIlan particularmente la autonomía de las nociones y las obligaciones positivas ${ }^{3}$.

Nos es dado pensar que, si se pretende que la propia Carta sea un instrumento de protección efectiva de los derechos sociales, se impone que el Comité Europeo de Derechos Sociales haga gala de ese mismo dinamismo interpretativo.

A primera vista, tal parece ser ya el caso. Diversas decisiones recientes, adoptadas en el marco del procedimiento de reclamaciones colectivas, pueden efectivamente corroborar la idea de una sensible convergencia en cuanto a los principios y métodos de interpretación entre la jurisprudencia del Comité y la del Tribunal. Así, ya encontramos desde la primera decisión adoptada en este marco - decisión Comisión Internacional de Juristas contra Portugal de 1999 relativa al trabajo infantil ${ }^{4}$ - la afirmación de la Carta como instrumento de derechos humanos. Este principio viene constantemente reiterado desde entonces, al igual que aquél otro según el cual la Carta atiende a la protección de derechos no teóricos sino efectivos. Más recientemente, en el asunto Federación internacional de ligas de derechos humanos contra Francia resuelto en 2004, el Comité invocó, como fundamento de su proceder interpretativo, el hecho de que la Carta es el complemento del $\mathrm{CEDH}$.

Mucho más que buenas intenciones, esos principios dan fundamento a una renovación de los métodos de interpretación y, de hecho, acentúan las exigencias de la Carta con respecto a los Estados Partes. Más precisamente, el principio de efectividad ha conducido en particular al Comité a imponer a dichos Estados obligaciones de resultado

3 Sobre estas cuestiones, será de utilidad para el lector acudir a SUDRE, F.: «À propos du dynamisme interprétatif de la Cour européenne des Droits de I'homme ", JCP - La semaine juridique, Ed. gén., 11 julio 2001, pp. 1365 ss.; léase asimismo el artículo precitado del mismo autor; y, sobre las obligaciones positivas, mi obra Les obligations positives en vertu de la Convention européenne des droits de l'homme, Ed. du Conseil de I'Europe, coll. Précis des Droits de I'homme, n. 7, 2006.

4 Las decisiones del Comité Europeo de Derechos Sociales se encuentran disponibles en el sitio Internet de la Carta Social, en la dirección siguiente: http://www.coe.int/T/F/Droits_de_l\%27Homme/Cse/. Para un análisis de ellas, me remito a mi crónica en la Revue trimestrielle des droits de l'homme: 2001/1035; 2003/113; 2004/225; 2005/673. 
allí donde hubiera podido pensarse que sobre ellos tan sólo pesaría una obligación de medios. Tal ha sido el caso, por ejemplo, en lo que atañe al trabajo infantil, en donde el compromiso de los Estados de «fijar en 15 años la edad mínima de admisión al trabajo" ha sido interpretado en el asunto Comisión Internacional de Juristas contra Portugal en el sentido de obligar a aquéllos, no sólo a adoptar medidas, sino también a obtener de hecho la supresión de las prácticas contrarias a dicha norma o, al menos, su sanción por el Derecho interno. Del mismo modo, en el asunto Autismo Europa contra Francia (2004), unas simples modificaciones legislativas han sido juzgadas insuficientes para satisfacer las exigencias del artículo 15.1, según el cual los Estados deben «tomar las medidas adecuadas para procurar a las personas minusválidas orientación, educación y formación profesional en el marco del régimen general, siempre que sea posible, o, en caso contrario, a través de instituciones especializadas, ya sean públicas o privadas». Se requiere, además, según el Comité Europeo de Derechos Sociales, que las medidas adoptadas apunten a conseguir el objetivo fijado por la Carta y que tales medidas produzcan los efectos susceptibles de ser esperados teniendo en cuenta dicho objetivo.

Los lazos estrechos entre Carta y CEDH, por su lado, han justificado la confluencia de la interpretación de algunas cláusulas de la primera, como la cláusula de no discriminación, con respecto a la de las disposiciones comparables del segundo, como a título de ejemplo puede observarse en el mencionado asunto Autismo Europa contra Francia. Más aún, esa relación estrecha ha servido de soporte a las tentativas del Comité tendentes a reducir los ángulos muertos de la Carta. Ha sido lo que ha sucedido, de manera particular, en lo atinente a la ampliación gradual del ámbito de aplicación personal de este instrumento. Sobre ello volveremos.

En estas condiciones, el desarrollo de una problemática común de protección de los derechos, ¿puede entonces a estas alturas presentarse como uno de los objetivos a alcanzar en el curso de la próxima década? Es lo que me inclino a pensar, por una razón esencial. El caso es que los avances que acaban de ser esbozados todavía se atisban lejanos en cuanto a su consolidación. Siguen siendo frágiles y se encuentran amenazados de ruina por todas partes.

De entrada, en el seno del propio Comité Europeo de Derechos Sociales. Si se observa atentamente su jurisprudencia desde la entrada en vigor de la Carta revisada, cabe entonces en algunas ocasiones plantearse si existe verdaderamente jurisprudencia, al menos si se repara en lo que este término puede sugerir en clave de estabilidad relativa y 
de claridad de soluciones. Las audacias y los retrocesos se van alternando a fuerza de mayorías fluctuantes, y muestran bien a las claras que nos hallamos lejos de un consenso, siquiera mínimo, a favor del dinamismo interpretativo preconizado.

Desde el exterior, más bien nos parece chocante la conjunción de lo que podríamos llamar "fuerzas de inercia». Ciertamente, éstas no son las únicas que "hacen la ley", en nuestro caso la jurisprudencia. Pero el hecho es que dichas fuerzas son poderosas. Aun a riesgo de simplificación, cabe identificar en esencia tres tendencias. En primer lugar, están los "integristas y fetichistas del texto", para quienes sólo existe el rigor jurídico en la interpretación literal e histórica, de suerte que cualquier alejamiento respecto a la letra de la Carta resulta ser una aproximación peligrosa. A continuación, están los "progresistas", que apuestan por una concepción que ve en la Carta una simple guía para las políticas sociales y, en consecuencia, postulan un control lo más leve posible, de modo que lo esencial para ellos sería que las situaciones nacionales vayan progresando, esto es, que respecto a un estadío anterior del Derecho y de la práctica internos se constate que los Estados realmente van caminando "en la buena dirección». Por último, están los "anti-fundamentalistas" quienes, sin duda por inclinación intelectual - se trata principalmente de especialistas de Derecho laboral-, ven en la Carta no tanto un instrumento de protección de los derechos humanos sino, ante todo, un convenio laboral, lo que no deja de proyectar consecuencias sobre la forma que tienen de entender y de aplicar el texto.

En suma, se tiene a veces la impresión, si se repasan las soluciones alcanzadas, de que la Carta no ha cambiado. Todo sucede como si el proceso de relanzamiento de la Carta no hubiera tenido lugar, y que los objetivos expresados por los textos salidos de ese relanzamiento, entre ellos "la indivisibilidad de todos los derechos humanos" (preámbulo de la Carta Social Europea revisada) y "la mejora de la efectividad de los derechos sociales" (preámbulo del Protocolo de 1995 sobre las reclamaciones colectivas), no debieran comportar consecuencia jurídica alguna.

Al hacer estas observaciones, evidentemente no estoy perdiendo de vista que el Comité Europeo de Derechos Sociales, desde luego más que otras instancias internacionales que tienen el cometido de decir el Derecho (más que el Tribunal Europeo de Derechos Humanos, por ejemplo), se encuentra sometido a fuertes presiones jurídicas y políticas. El hecho de que el proceso de ratificación de la Carta revisada y del Protocolo sobre las reclamaciones colectivas todavía esté en curso es de hecho un dato a tener en cuenta. La necesidad de generar en torno a 
las decisiones adoptadas un amplio consenso político-jurídico que favorezca la buena ejecución de ellas es otro dato a tener en cuenta. En fin, debe mencionarse la obligación, la más compartida y que se desprende de los textos, de que las decisiones del Comité sean construidas y fundadas en Derecho.

Pero, si esas presiones pueden explicar algunos de los rasgos señalados con anterioridad, tampoco cabe exagerar su alcance. Estos actores no explican todo. Los hábitos intelectuales bien podrían tener una fuerza equiparable. Y cabría igualmente plantearse el papel jugado por los criterios y modalidades actuales de designación de los miembros del Comité. Sea como fuere, dichas presiones no vedan cualquier tipo de avance jurisprudencial, ni pueden justificar un conservadurismo exacerbado.

El caso es que, y se convendrá en ello fácilmente, la fragilidad de los progresos operados a lo largo de esta década por el Comité Europeo de Derechos Sociales no se deben solamente a la incoherencia de su jurisprudencia o a las correlaciones de fuerzas en el seno de dicha instancia. A ello contribuye asimismo en cierta medida la actitud del Comité de Ministros. Ya sabemos que, en su condición de órgano de la Carta, dicho Comité, en particular en el marco del procedimiento de reclamaciones colectivas, debe levantar acta, mediante una resolución, de las decisiones del Comité Europeo de Derechos Sociales y, en caso de haberse constatado una violación, recomendar al Estado afectado las medidas que estime apropiadas con el objeto de que la situación interna llegue a ser conforme a la Carta. No volveremos sobre el modo equívoco en que el órgano ministerial ha venido desempeñando hasta la fecha dicha misión ${ }^{5}$. Lo esencial aquí y ahora radica en subrayar que el Comité de Ministros parece haber interpretado su misión, no sólo en el sentido de verse obligado a velar por la correcta ejecución interna de las decisiones del Comité Europeo de Derechos Sociales, sino asimismo en el sentido de entenderse habilitado para reexaminar, como si se tratase de una instancia de apelación, las decisiones adoptadas por este último. Esta interpretación absolutamente particular y rechazable de los textos le ha llevado en el primer asunto sobre las 35 horas (Confederación francesa de directivos-Confederación general de ejecutivos contra Francia, 2000), a invalidar parcialmente la decisión condenatoria para Francia. Semejante práctica, sin lugar a dudas, no hace sino comprometer la autoridad de las apreciaciones jurídicas del Comité Europeo de Derechos Sociales y socavar la legitimidad de este órgano. Así, es

${ }^{5}$ Sobre esta cuestión, véanse mis crónicas precitadas. 
deseable que dicha práctica no se perpetúe. La posición del órgano ministerial sigue siendo, a este respecto, ambigua. En efecto, si bien es verdad que se ha abstenido, tras ese primer asunto sobre las 35 horas, de apreciar jurídicamente las decisiones del Comité Europeo de Derechos Sociales, no es menos cierto que también se ha guardado, en el segundo asunto sobre las 35 horas (Confederación francesa de directivos-Confederación general de ejecutivos contra Francia, 2003), de suscribir formalmente la interpretación efectuada por este último Comité respecto a las disposiciones pertinentes del Protocolo de 1995 y de las que se desprende que sólo es competente el Comité Europeo de Derechos Sociales para apreciar la conformidad con la Carta de las legislaciones, reglamentos y prácticas nacionales. Ya es tiempo de acabar con este equívoco.

Suponiendo que las condiciones evocadas se acaben verificando para un progreso duradero de la jurisprudencia, esto es, que el Comité Europeo de Derechos Sociales acepte dotar plenamente de sentido y efecto a los fines que establece la Carta y que, paralelamente, el Comité de Ministros adopte una actitud más compatible con el desarrollo de la competencia el Comité Europeo de Derechos Sociales, restará por saber en qué disposiciones de la Carta revisada debería basarse prioritariamente la obra interpretativa.

\section{Las evoluciones deseables}

La construcción de una problemática común sobre la protección de los derechos humanos debería implicar que todas las disposiciones sustanciales de la Carta fueran interpretadas siguiendo la perspectiva anunciada por algunas decisiones recientes, a saber, la protección efectiva de los derechos. No procede en esta sede ir más allá de esta proposición general. Corresponde al Comité Europeo de Derechos Sociales extraer las correspondientes consecuencias prácticas en cada asunto que le sea sometido. La única cuestión que se plantea aquí es, como se ha visto, la referente a la determinación del Comité de adoptar y mantener en el tiempo una política jurisprudencial dinámica, que se adecue a la letra y al espíritu —entendiendo por tal la naturaleza y la finalidadde la Carta.

Por tanto, es evidente que algunas cláusulas de la Carta, en razón de su misma naturaleza, apelan al Comité para que éste lleve a cabo una acción interpretativa más incisiva: se trata precisamente de aquéllas cuya incompatibilidad con una problemática de los derechos fundamentales es flagrante. Estoy pensando aquí en el Anexo de la Carta y 
en su artículo I, es decir, en las disposiciones que definen restrictivamente el ámbito de aplicación personal de este instrumento, al excluir a los extranjeros y al legitimar las "violaciones menores" de los derechos garantizados, respectivamente.

Ya he tenido ocasión de abordar con detalle esas disposiciones y su carácter inicuo en un instrumento de protección de derechos humanos ${ }^{6}$. Sin embargo, no resulta inútil volver sobre ello brevemente. En cuanto al Anexo, debe recordarse que éste prevé que las personas protegidas por la Carta "sólo comprenden a los extranjeros que, siendo nacionales de otras Partes, residan legalmente o trabajen habitualmente dentro del territorio de la Parte interesada". En lo que atañe al artículo l, éste dispone en su apartado 2 que los compromisos derivados de algunas disposiciones del instrumento europeo se considerarán cumplidas «siempre que las disposiciones se apliquen (...) a la gran mayoría de los trabajadores interesados", lo que había conducido inicialmente al Comité Europeo de Derechos Sociales a considerar que bastaba, para cumplir con las exigencias de las disposiciones correspondientes de la Carta, que las medidas internas de puesta en práctica fueran aplicadas al $80 \%$ al menos de los trabajadores.

El Comité Europeo de Derechos Sociales ha intentado en los últimos años reducir los "ángulos muertos" creados por estas dos disposiciones. Soy de los que piensan que dicho órgano ha sabido, en esta materia, establecer un justo equilibrio entre el respeto de la letra de estas cláusulas de exclusión y el espíritu de la Carta, que ante todo es un convenio de derechos humanos, y que ese espíritu impone proteger a toda persona que se encuentre bajo la jurisdicción de los Estados Partes. En lo que concierne a la cláusula del Anexo, la solución alcanzada en el asunto Autismo-Europa contra Francia (2004) ha consistido en descartar su aplicación cuando ésta sea incompatible con el debido respeto a la vida y a la dignidad humana. Así, se procede al tiempo a confirmar la validez de dicha cláusula y a promover la aplicabilidad de la Carta a los extranjeros en circunstancias excepcionales ${ }^{7}$. Lo mismo ha

6 Sobre este punto, me permito remitir al lector a mis estudios anteriores: "The material impact of the jurisprudence of the European Committee of social rights", en el colectivo G. De Burca \& B. De Witte, Social rights in Europe, Oxford University Press, 2005, pp. 89 ss.; así como "La Charte sociale européenne et la promotion des droits sociaux", en el colectivo Les droits sociaux entre droits nationaux et droit européen, Actas del coloquio de Aix, pendientes de publicación en la editorial Bruylant.

7 Para un análisis en profundidad de esta decisión y sus implicaciones, véase mi estudio: La Charte sociale et la protection des migrants en situation irrégulière, Me- 
sucedido con el artículo I. En efecto, de la decisión adoptada en el primer asunto sobre las 35 horas se desprende que dicha disposición no autoriza a los Estados a excluir deliberadamente de la aplicación de la Carta a grupos de personas. Dicho de otro modo, acudiendo a los términos de la propia decisión, su aplicación «no puede conducir a una situación en la que un número elevado de personas, que constituya una categoría determinada, sea deliberadamente excluida de la aplicación de una disposición normativa».

En semejante contexto, el reto de la próxima década habría de ser no tanto la interpretación misma de dichas cláusulas sino el dotar de estabilidad a la jurisprudencia sobre la materia y a su desarrollo.

Es bajo estas condiciones como cabe esperar que la Carta se aproxime al estándar del CEDH. Pero no nos Ilamemos a engaño. La Carta sólo estará en condiciones de alcanzar efectivamente ese estándar a partir del momento en que las dos cláusulas singulares reseñadas con anterioridad, cuya problemática —recordémoslo- es extraña a la problemática de los derechos humanos, queden simple y llanamente derogadas y, en consecuencia, la protección establecida por la Carta puede beneficiar a cualquier persona que se encuentre bajo la jurisdicción de los Estados Partes.

\section{EL ESTABLECIMIENTO DE ESPECÍFICAS ARTICULACIONES INSTITUCIONALES Y PROCESALES ENTRE LA CARTA Y EL CEDH}

Como podrá colegirse de lo expuesto hasta ahora, el llamamiento al desarrollo de una problemática común referente a la protección de los derechos humanos es, de hecho, una invitación a la profundización de dinámicas que ya existen. No sucede lo mismo en lo que concierne a la proposición que ahora nos ocupa. En efecto, debe quedar sentado que, hasta el presente, no existe pasarela alguna de carácter institucional o procedimental entre la Carta y el CEDH. Se trata, por tanto, de crearlas.

La razón esencial por la cual semejante evolución puede ser deseable radica en que el sistema existente, en el cual cada órgano de control tiene o no en cuenta e interpreta el otro instrumento según le conviene, parece haber llegado a sus límites. Ello podría incluso considerarse pernicioso, en la medida en que puede conducir a situaciones carentes

morandum, Assemblée parlementaire du Conseil de l'Europe, Commission des migrations, des réfugiés et des populations, doc. AS/Mig/lnf (2005) 17, 28 sept. 2005. 
de protección. Los ciudadanos europeos serían, por consiguiente, las primeras "víctimas" de esa práctica, lo cual no resulta admisible, por más que éstos sólo representen una ínfima minoría.

La proposición que nos ocupa no tiene nada de inédito en el fondo. En el momento de la elaboración de la Carta, ya se había afrontado la posibilidad de concebir el mecanismo internacional de control de tal suerte que éste no quedara totalmente separado del establecido en el CEDH. El medio entonces imaginado, a tenor del anteproyecto de Carta fechado el 19 de abril de 1957, era institucional: la Comisión europea de derechos humanos debía ser un órgano común a los dos sistemas. Estas propuestas, como es sabido, no triunfaron. La problemática que subyacía en dichas propuestas merecería sin embargo ser reactivada.

La cooperación entre los dos sistemas convencionales perseguiría un doble objetivo: de un lado, luchar contra la pérdida de recursos y, por tanto, contra la carencia de protección; de otro lado, reducir los riesgos de conflictos interpretativos $y$, por ende, de divergencia de jurisprudencias.

\section{Soluciones para luchar contra la pérdida de recursos}

El desconocimiento de la Carta Social y de su dispositivo de control e, inversamente, la gran difusión del CEDH conducen naturalmente a los demandantes a dirigir sus demandas al Tribunal Europeo de Derechos Humanos antes que al Comité Europeo de Derechos Sociales. Lo cual parece ser de lo más normal, en tanto en cuanto estén en juego derechos garantizados por el Convenio de 1950. Pero ha de repararse asimismo en que a veces se formulan ante la Secretaría del Tribunal recursos tendentes a la protección de derechos sociales, y respecto de los cuales el Tribunal muestra más bien una tendencia a cerrarles el paso a su labor pretoriana. Así, en 1999, en el caso Paneenko contra Letonia, declaró manifiestamente infundada, y consecuentemente inadmisible, la demanda que se había formulado ante él en la que se alegaba una violación del derecho al trabajo. Lo mismo sucedió en 2002 en el caso Salvetti contra Italia a propósito del derecho a la asistencia médica gratuita. Y, cuando la demanda llega pese a todo a superar el filtro de la admisibilidad, todavía existen grandes posibilidades de que, como ocurrió en el asunto Botta contra Italia (1998), la disposición invocada del Convenio sea declarada inaplicable. Podría argüirse que, desde esta perspectiva, habría pocos casos para justificar una reforma. Pero, al margen de algunas demandas conocidas, ¿qué sabemos respecto a las demás? ¿Cuántas de éstas no habrán podido franquear el filtro pre- 
vio de la Secretaría del Tribunal por haber sido consideradas manifiestamente infundadas o sin objeto? Por lo demás, sería interesante conocer las cifras para comprobar con precisión la demanda de derechos sociales en Europa.

En lo anterior seguramente no quepa entrever fatalidad alguna. Como ha demostrado brillantemente el Profesor Sudre en un ejercicio de "jurisprudencia-ficción ${ }^{8}$, sería perfectamente concebible, a tenor de las técnicas y métodos de interpretación que ha desarrollado, que el Tribunal Europeo acogiera tales recursos y asegurara la protección de los derechos que constituyen su objeto. Se llegaría entonces a una situación en la que lo esencial de los derechos garantizados por la Carta Social podría beneficiarse de una protección suplementaria, en este caso judicial e individualizada. Por otra parte, cabrá observar, dicho sea de paso, que la verificación de semejante virtualidad haría nacer, si los mecanismos propios de la Carta se mantuvieran como tales, una necesidad todavía mayor de coordinación. Más precisamente, es obvio que hoy por hoy el Tribunal Europeo no se entiende comprometido por esta vía. Con ello retornamos a nuestro punto de partida, a la situación que prevalece actualmente y en la cual los recursos seguirán con la tendencia a inscribirse en el marco del Convenio antes que en el de la Carta; una situación en la cual los demandantes acudirán por tanto, con la mejor buena fe, a una vía sin salida, para la protección de un derecho que, no obstante, les garantiza un tratado del Consejo de Europa.

Hay quien objetará que el riesgo aquí evocado sólo es teórico; que el demandante ante el Tribunal Europeo de Derechos Humanos no es el mismo que ante el Comité Europeo de Derechos Sociales. En un caso, ante el Tribunal, la víctima es la persona, en la mayor parte de los casos un individuo, que puede ejercer el derecho a un recurso; en el otro, ante el Comité Europeo de Derechos Sociales, se trata de una organización que no necesita acreditar un perjuicio sufrido por ella misma. La objeción parece seria. Pero puede quedar fácilmente descartada sobre la base de dos argumentos:

En primer lugar, debe hacerse notar que lo "colectivo" sólo se opone aquí a lo "individual» hasta un cierto punto. Por una parte, los demandantes en el sistema de reclamaciones son personas individualizadas y sólo se diferencian de las organizaciones no gubernamentales admitidas ante el Tribunal Europeo de Derechos Humanos por su relación con las violaciones alegadas - la calidad de "víctima" exigida a estas últimas-; por otra parte, si las reclamaciones según el Protocolo de 1995 deben ver-

${ }^{8}$ Artículo precitado, Rev. trim. dr. h., 2003, pp. 755 ss. 
sar sobre "situaciones" y, por tanto, referirse a violaciones generales, es preciso advertir que éstas pueden perfectamente afectar a personas identificables o, dicho de otro modo, según la terminología del $\mathrm{CEDH}$, un "grupo de particulares". Es decir, que en la teoría, la distancia que separa el procedimiento de reclamaciones colectivas del procedimiento de recursos individuales tal vez no sea tan grande.

Pero todavía lo es menos en la práctica. Cabe observar, en efecto, y en ello radica el segundo argumento, que en los asuntos sometidos al Comité Europeo de Derechos Sociales se combinan frecuentemente recursos individuales y colectivos. Así, no es extraño, y ha sido el caso en todos los asuntos franceses, que la reclamación colectiva tome el relevo de procedimientos iniciados por particulares ante las jurisdicciones nacionales. Es, por lo demás, esta circunstancia la que ha conducido al Comité a recordar, tal como lo hizo en el caso Sindicato de profesores agregados de la enseñanza superior contra Francia (2004, admisibilidad), que el Protocolo de 1995 no subordina la presentación de reclamaciones al agotamiento de los recursos internos. Por lo demás, resulta revelador que en varios asuntos, las organizaciones reclamantes hayan planteado, en caso de no prosperar la reclamación y tras el agotamiento de los recursos nacionales, la posibilidad de acudir al Tribunal Europeo de Derechos Humanos. El desfase temporal que existe necesariamente entre los dos tipos de procedimientos, y que tiene que ver como se ha visto con la condición del agotamiento de las vías nacionales de recurso en el caso del CEDH, explica, por sí sola, que todavía no hayamos asistido a semejante combinación. Pero a buen seguro que esa combinación se concretará en un futuro próximo, puede que hasta de una manera positiva pues, como demostraremos más tarde, aun teniendo en cuenta la reticencia del Tribunal Europeo de Derechos Humanos con respecto a los derechos sociales, no están ausentes los puntos de intersección entre el Convenio y la Carta.

Retomando el discurso principal cabe advertir que, si se quiere luchar eficazmente contra el riesgo de pérdida de recursos en materia de derechos sociales o, dicho de otro modo, si se quiere que dichos recursos sean ejercidos de forma útil y, por ende, que los derechos sociales sean protegidos con mayor eficacia, parece que debe adoptarse un cierto número de medidas.

La primera de ellas podría consistir en establecer en el seno del Consejo de Europa, una estructura operativa de conexión entre la Carta y el Convenio cuya función sería informar a los demandantes potenciales sobre las posibilidades que les ofrecen los instrumentos de la organización y, en su caso, aconsejarles y orientar su acción hacia el 
procedimiento más apropiado. La existencia de semejante estructura, a poco que desempeñara bien su función, podría contribuir, en lo que atañe a las demandas tendentes a la protección de los derechos sociales, a reducir la saturación del Tribunal Europeo en beneficio del Comité Europeo de Derechos Sociales.

Ello no obstante, hay que convenir en que esta solución no está en condiciones de resolver todas las dificultades. No debe olvidarse que la puerta, tanto de un procedimiento como del otro, todavía permanece cerrada a algunas demandas: aquellas que invocan un derecho garantizado por la Carta Social y excluido del ámbito de aplicación del CEDH y que, por añadidura, son presentadas por la propia víctima. En la hipótesis de que dichos recursos se multiplicaran, y en la medida en que éstos revelaran la existencia de una situación nacional preocupante a la luz de la Carta, ¿no cabría afrontar que se encomendare a una autoridad del Consejo de Europa, por ejemplo el Comisario para los derechos humanos, la posibilidad de recurrir ante el Comité Europeo de Derechos Sociales, sea en el marco del procedimiento de reclamaciones, sea en el del procedimiento de informes?

\section{Mecanismos para limitar los riesgos de conflictos interpretativos}

Si la necesidad de pasarelas entre la Carta y el Convenio es perceptible, es ante todo porque ya de hecho existen interferencias entre los dos textos - o, mejor aún, entre los dos sistemas de instrumentos-, interferencias que la jurisprudencia ha venido a acentuar.

De hecho, algunos derechos se encuentran reconocidos en los dos sistemas. Es el caso de la prohibición del trabajo forzado, explícitamente enunciado en el artículo $4 \mathrm{CEDH}$ y en el artículo 1.2 de la Carta según la interpretación del Comité Europeo de Derechos Sociales; del derecho de sindicación consagrado en el artículo 11 del primer texto y el artículo 5 del segundo texto; de los derechos a la seguridad social y a la asistencia social, protegidos por los artículos 12 y 13 de la Carta y que el Tribunal ha deducido del derecho patrimonial (artículo $1 .^{\circ}$ del primer Protocolo adicional al Convenio). Debe mencionarse igualmente una cierta orientación de la Carta efectuada por el Comité que tiende a extraer de algunos de los derechos garantizados por el CEDH (tales como el derecho a la vida privada o familiar, la prohibición de malos tratos o el derecho a la libertad de expresión) prolongaciones necesarias de los derechos sociales ${ }^{9}$.

\footnotetext{
9 Véanse mis estudios citados en nota 6.
} 
Esas interferencias se verán seguramente multiplicadas de manera exponencial con la entrada en vigor del Protocolo n. ${ }^{\circ} 12$ al Convenio ${ }^{10}$. Éste, como es sabido, prohíbe toda discriminación en el ejercicio de derechos, tanto si estos últimos están consagrados por el propio Convenio, como si lo están por otros textos europeos o internacionales o por el Derecho interno. Por lo tanto, los derechos garantizados por la Carta Social, sin excepción, entrarán en el ámbito de aplicación del Protocolo y el Tribunal Europeo de Derechos Humanos será competente para conocer de las alegaciones de discriminación en el disfrute de aquéllos. Ahora bien, la propia Carta ya enuncia semejante prohibición en lo que concierne a los derechos por ella garantizados. Luego, podría suscitarse el temor de que, elaborada por dos instancias con enfoques no necesariamente convergentes, la jurisprudencia relativa a la Carta pueda llegar a ser incoherente en el mejor de los casos, o contradictoria en el peor de ellos. Por otra parte, semejante resultado afectaría no sólo a la comprensión del principio de no discriminación, sino también a la de las disposiciones sustanciales de la Carta pues, antes de determinar si existe o no discriminación, debe previamente definirse la consistencia y el perímetro del derecho de referencia.

Teniendo en mente ese riesgo, y con el fin de ponerle remedio, algunos expresan entre sus deseos el de establecer, entre el Tribunal y el Comité, un procedimiento de cuestión prejudicial. Se trataría de que, en cada ocasión en la que el derecho objeto del recurso fuera uno de los consagrados por la Carta, el Tribunal Europeo debería aplazar su pronunciamiento e interrogar previamente al Comité Europeo de Derechos Sociales, el cual procedería entonces a efectuar una interpretación que la jurisdicción europea no tendría más que asumir. El modelo en el que se inspiran estas sugerencias parece ser el de la cuestión prejudicial existente en el orden jurídico comunitario.

Basta con apenas haber enunciado dicha idea para poner en entredicho su inadaptación al problema planteado. Aunque en Derecho comunitario se la tenga por un procedimiento de cooperación entre dos jueces -0 , tras algunas innovaciones introducidas por el Tratado de Ámsterdam, entre dos autoridades de las cuales una es jurisdiccional-, el procedimiento de la cuestión prejudicial no instaura una relación de tipo piramidal entre dos autoridades que pertenezcan a un mismo orden jurídico. Ese carácter deriva principalmente del monopolio, reconocido a la instancia ante la que se formula la cuestión, en

10 Véase sobre este tema el artículo precitado de F. Sudre, Rev. trim. dr. h., 2003, pp. 755 ss. 
materia de interpretación (y/o de apreciación de la validez), y su justificación principal reside en la exigencia de coherencia, e incluso de unidad de aplicación del Derecho en un orden jurídico dado.

Ninguna de las condiciones evocadas se da en el presente caso. Carta y Convenio son dos instrumentos autónomos de Derecho internacional que por sí solos determinan la competencia de los órganos que establecen. No hay lugar, en semejante contexto, para relaciones del tipo de las descritas, por lo que sólo cabría concebir que uno de los órganos renuncie a una parte de su competencia - lo que debería hacer el Tribunal Europeo- en beneficio del otro, cuya existencia y competencia se basan en otro texto. A decir verdad, las únicas fórmulas concebibles en el estado actual del Derecho del Consejo de Europa son de coordinación o, en el mejor de los casos, de armonización voluntaria de jurisprudencias. Pero hace falta que existan, lo que todavía no sucede hoy en día.

Una de las soluciones en disposición de permitir la prevención del riesgo de jurisprudencias divergentes podría consistir en prever una posibilidad de intervención del Comité Europeo de Derechos Sociales en el marco del procedimiento ante el Tribunal Europeo e, inversamente, la posibilidad de que éste interviniere en el marco del procedimiento de reclamaciones colectivas. Esto sería posible a través del mecanismo de la intervención de terceros que existe tanto en uno como en otro contexto procedimental. Semejante facultad estaría reservada a los casos en que el asunto sometido a examen presentare un interés desde el punto de la Carta o del Convenio, y la posición adoptada por esa intervención del órgano tercero no vinculare al órgano competente. Nótese que dicha innovación no implicaría necesariamente una reforma del CEDH. Una interpretación apropiada de la noción de "personne intéressée autre que le requérant $X X X$ " a la que se refiere el artículo 36 de este texto convencional debería ser suficiente. En cuanto al reglamento del Comité Europeo de Derechos Sociales, su artículo 32 deberá ser reformado necesariamente para incluir al Tribunal Europeo de Derechos Humanos en la lista de los terceros coadyuvantes, lo cual no debería plantear en principio mayores dificultades, en la medida en que el Comité es dueño de su reglamento.

A lo anterior cabría añadir que dicho mecanismo sólo cumpliría plenamente su función en un contexto de cooperación confiada entre los dos órganos, pues debe repararse en que una intervención de terceros sólo se pone en marcha a iniciativa del Presidente de la instancia competente, y las observaciones escritas u orales que se efectúen se dejan a la discreción de los miembros de dicha instancia en lo que 
atañe a su toma en consideración. La eficacia del mecanismo dependería así de la buena voluntad de los miembros del Tribunal Europeo y del Comité Europeo de Derechos Sociales, así como del clima que sepan instaurar entre ellos. Sin duda alguna, a ello contribuiría la organización de un diálogo institucionalizado entre estos órganos que se inspirara en el que ya funciona desde hace muchos años entre el Tribunal Europeo de Derechos Humanos y el Tribunal de Justicia de las Comunidades Europeas.

\section{REFLEXIONES FINALES}

Llegados a este punto, dejo planteadas así algunas de mis propuestas. Éstas no pretenden agotar el tema. Podrían ser objeto de ampliación en otros ámbitos. Pero lo que importa es, sobre todo, recordar el espíritu con el que son efectuadas y con el cual, llegado el caso, podrían ser puestas en práctica.

Primo, diseñar nexos entre el sistema del CEDH y el de la Carta no significa necesariamente que deba asegurarse una identidad perfecta entre ellos. Si en lo que se ha insistido en esta contribución ha sido sobre todo en la necesidad de elevar el segundo sistema al nivel del primero, también es verdad que algunos aspectos de la Carta Social manifiestan una originalidad positiva y deben ser salvaguardados. Podrían incluso inspirar a aquellos que están llamados a reflexionar sobre el desarrollo futuro del CEDH. Estoy pensando aquí especialmente en el mecanismo de control y en la baza o el éxito que podría representar la combinación de un procedimiento de recurso con un sistema de informes periódicos que versa sobre la totalidad de los compromisos adquiridos por todos los Estados Partes.

Secundo et in fine, hacer avanzar pragmáticamente la Carta es deseable, pero a condición de que ello no se utilice como pretexto para operar una transferencia subrepticia de poderes de los Estados en materia de reforma de los tratados a órganos que no ostentan título alguno para ejercerlos. Es decir, el pragmatismo del progreso debe tener sus propios límites. En caso contrario, dicho pragmatismo chocaría con la desconfianza legítima de los Estados. Dicho en otros términos y en clave positiva, la respuesta a las dificultades y lagunas evocadas más arriba sólo podrá venir en última instancia de la mano de una reforma en toda regla de los dos tratados afectados. Así, las autoridades responsables encontrarían la inspiración adecuada para acometer desde este momento la reflexión, pero también la acción, en la perspectiva expuesta. 
Title:

"European Social Charter and European Convention on Human Rights: Perspectives for the next decade».

\section{Summary:}

I. INTRODUCTORY CONSIDERATIONS. II. THE DEVELOPMENT OF A COMMON PROBLEMATICS OF GUARANTEE: 1. The way: the interpretation of the Charter. 2. The desirable evolutions. III. THE ESTABLISHMENT OF SPECIFIC INSTITUTIONAL AND PROCEDURAL MECHANISMS BETWEEN THE CHARTER AND THE ECHR: 1. Solutions to face up to the loss of remedies. 2. Mechanisms to limit the risks of interpretive conflicts. IV. FINAL REFLECTIONS

\section{Resumen:}

La contribución parte de una premisa: la caracterización de la Carta Social Europea como un complemento del Convenio Europeo de Derechos Humanos no es un obstáculo para considerar la Carta Social desde una perspectiva comparada y complementaria de ambos instrumentos europeos. Desde este punto de vista, el principio de indivisibilidad de todos los derechos humanos juega un papel fundamental. En este sentido, el mayor conocimiento y éxito del Convenio Europeo no debe hacer olvidar que la Carta Social constituye el instrumento de democracia social por excelencia del Consejo de Europa, un instrumento específico de derechos fundamentale sociales que, en este preciso ámbito, puede ser más efectivo que el Convenio Europeo. Con este enfoque, el trabajo del Profesor Akandji-Kombé pone el énfasis en el principio más favorable a la protección de los derechos fundamentales, de manera que propone una serie de mecanismos de articulación institucional y procesal entre la Carta y el Convenio para, de un lado, evitar la formulación de recursos inútiles que no conduzcan a la solución más favorable para la dignidad humana y, de otro lado, limitar asimismo los riesgos de conflictos interpretativos entre el Comité Europeo de Derechos Sociales y el Tribunal Europeo de Derechos Humanos. Se trata, en definitiva, de una apuesta por la consolidación del Derecho Europeo de los Derechos Humanos, en donde la evolución de la Carta Social no debe hacerse depender exclusivamente del pragmatismo interpretativo del Comité Europeo de Derechos Sociales, sino asimismo del compromiso de los Estados miembros del Consejo de Europa para dotar de legitimidad a esa evolución a través de la oportuna reforma de la Carta Social. 


\begin{abstract}
:
This paper poses this premise: the characterization of the European Social Charter as a complement to the European Convention on Human Rights is not an obstacle, as the Social Charter can be considered from a compared and complementary perspective of both European instruments. From this point of view, the principle of indivisibility of all human rights plays a fundamental role. With respect to this, the greater knowledge and success of the European Convention must not forget that the Social Charter constitutes the paradigmatic instrument of social democracy within the Council of Europe, that is to say, a specific instrument of fundamental social rights that, in this precise area, can be more effective than the European Convention. With this approach, Professor Akandji-Kombé argues that the emphasis on the principle of most favorable protection of fundamental rights leads to the adoption of a series of mechanisms of institutional and procedural articulation between the Charter and the Convention in order to, avoid the formulation of useless requests that do not lead to the most favorable solutions for human dignity and, on the other hand, limit the risks of interpretive conflicts between the European Committee of Social Rights and the European Court of Human Rights. It is a question, definitively, in support of the consolidation of European Human Rights Law, where the evolution of the Social Charter must not depend exclusively on the interpretive pragmatism of the European Committee of Social Rights, but likewise of the commitment of the members states of the Council of Europe to provide with legitimacy to this evolution across the opportune reform of the Social Charter.
\end{abstract}

\title{
Palabras clave
}

Indivisibilidad de derechos fundamentales, complementariedad de tratados europeos, dinamismo del Comité Europeo de Derechos Sociales, evolución interpretativa y reforma de Carta Social Europea, articulación con Convenio Europeo de Derechos Humanos.

\section{Key words:}

Indivisibility of fundamental rights, complementarity of European treaties, dynamism of the European Committee of Social Rights, interpretive evolution and reform of the European Social Charter, articulation with the European Convention on Human rights. 